\title{
Erratum: Microscopic calculations based on chiral two- and three-nucleon forces for proton- and ${ }^{4} \mathrm{He}$-nucleus scattering [Phys. Rev. C 92, 024618 (2015)]
}

\author{
M. Toyokawa, M. Yahiro, T. Matsumoto, K. Minomo, K. Ogata, and M. Kohno \\ (Received 6 June 2017; published 27 November 2017)
}

DOI: 10.1103/PhysRevC.96.059905

As reported in the Erratum [1], several errors were found in the program code for the nuclear-matter calculation reported in Ref. [2]. Changes in the $G$ matrices in nuclear matter including the three-nucleon force (3NF) effects affect the results of subsequent applications of them to the description of nucleon-nucleus and ${ }^{4} \mathrm{He}$-nucleus scatterings reported in the original paper. We present corrected results in this Erratum. Our conclusion for the $3 \mathrm{NF}$ effects in the present elastic scattering does not change qualitatively.

Concerning the original paper, the corrected single-particle potential energies for each spin-isospin channel are shown in Fig. 2, which replaces Fig. 2 in the original paper. The $3 \mathrm{NF}$ effects become weaker except for the real part in the ${ }^{3} \mathrm{O}$ channel. Because the nucleon-nucleus elastic scattering

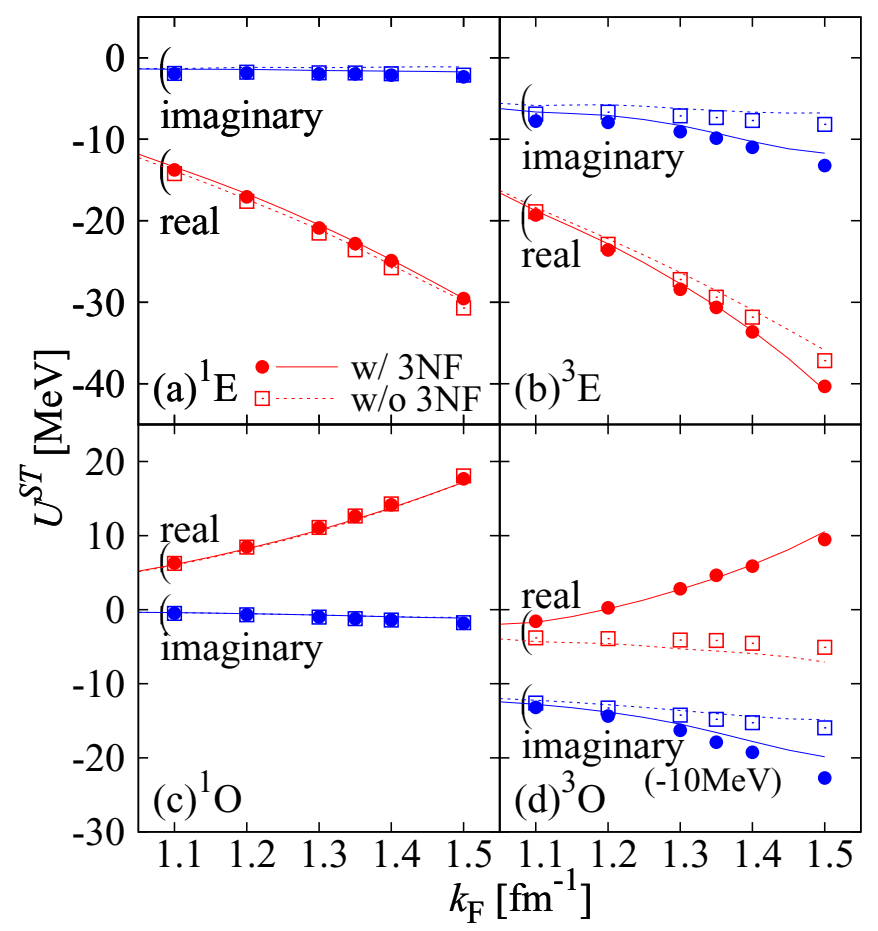

FIG. 2. Revised version of Fig. 2 in the original paper. $k_{\mathrm{F}}$ dependence of the optical model potential from each spin and isospin channel at $E_{\text {in }}=65 \mathrm{MeV}$ for (a) singlet-even $\left({ }^{1} \mathrm{E}\right)$, (b) triplet-even $\left({ }^{3} \mathrm{E}\right)$, (c) singlet-odd $\left({ }^{1} \mathrm{O}\right)$, and (d) triplet-odd $\left({ }^{3} \mathrm{O}\right)$. The squares (circles) mean the results of the original chiral $g$ matrix with (without) $3 N F$. The solid (dashed) lines represent the results of the Gaussian chiral $g$ matrix with (without) $3 \mathrm{NF}$. For ${ }^{3} \mathrm{O}$, the imaginary part is shifted down by $10 \mathrm{MeV}$. mainly takes place at the low-density surface region, $3 \mathrm{NF}$ effects on the scattering are still rather small. It is noted that the surface dominance is reinforced by the increase in the imaginary strength of the optical model potential from the $3 \mathrm{NF}$ effects. The revised angular distributions of proton scattering on ${ }^{40} \mathrm{Ca},{ }^{58} \mathrm{Ni}$, and ${ }^{208} \mathrm{~Pb}$ at $65 \mathrm{MeV}$ are shown in Fig. 3 as the replacement of Fig. 3 in the original paper. In the process of simulating calculated $G$ matrices in a three-range Gaussian

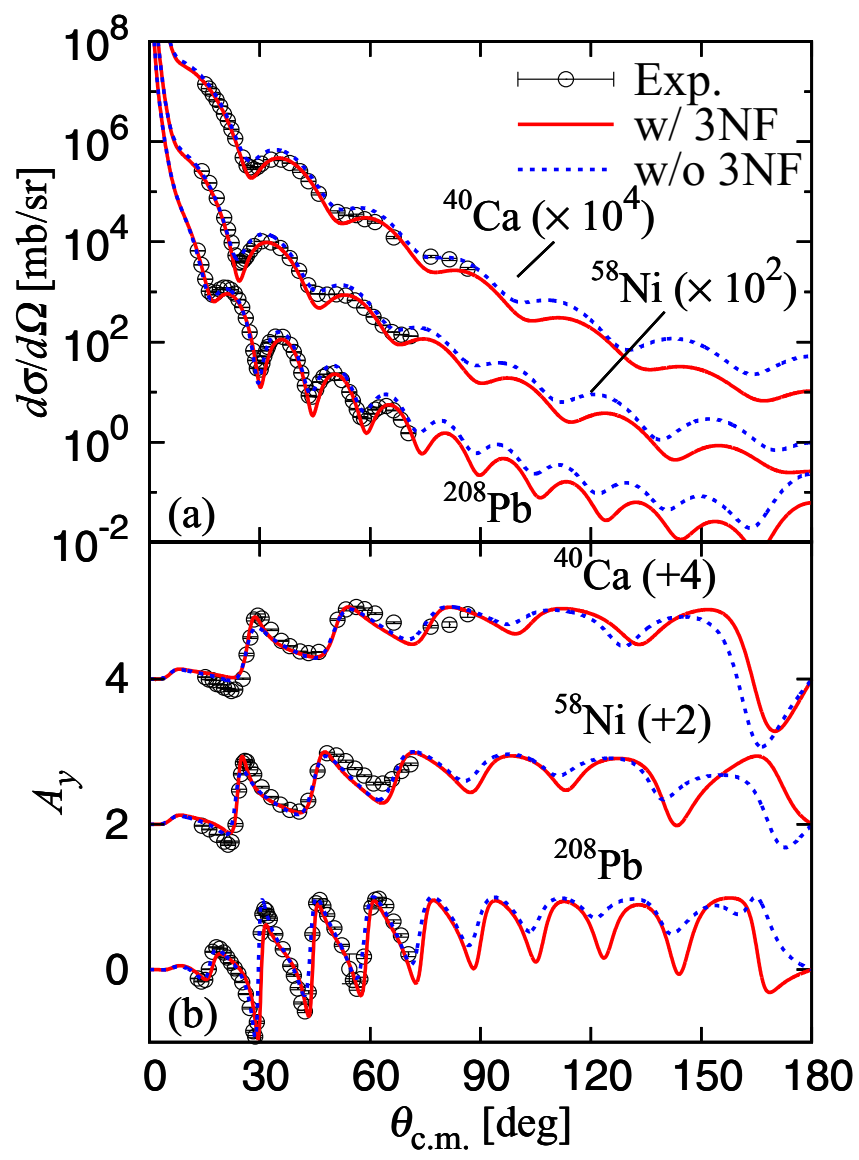

FIG. 3. Revised version of Fig. 3 in the original paper. Angular distribution of (a) differential cross sections and (b) vector analyzing powers for proton elastic scattering at $65 \mathrm{MeV}$. The solid (dashed) lines denote the results of the chiral $g$ matrix with (without) $3 \mathrm{NF}$ effects. Each cross section is multiplied by the factor shown in the figure, whereas each vector analyzing power is shifted up by the number shown in the figure. Experimental data are taken from Ref. [3]. 
form, it was very difficult to obtain reasonable parameters for the ${ }^{3} \mathrm{E}$ spin-orbit component. Because the strength is small and moreover large cancellation in the folding potential is expected between direct and exchange contributions, this component is ignored in the present calculations.
It is mentioned that the desirable $3 \mathrm{NF}$ effects observed in the ${ }^{4} \mathrm{He}$ scattering at $72 \mathrm{MeV} /$ nucleon from ${ }^{58} \mathrm{Ni}$ and ${ }^{208} \mathrm{~Pb}$ in Fig. 4 of the original paper are found to be scarcely affected by the revision.
[1] M. Kohno, this issue, Phys. Rev. C 96, 059903 (2017).

[2] M. Kohno, Phys. Rev. C 88, 064005 (2013).
[3] H. Sakaguchi et al., Phys. Lett. B 89, 40 (1979); 99, 92 (1981). 